\title{
Frau F. sorgt zunehmend für sich selbst
}

\section{Prozessuale strukturbezogene Diagnostik in der Integrativen Gestalttherapie (IG)}

\author{
Markus Hochgerner • Petra Klampfl
}

Online publiziert: 9. Juni 2020

(C) Der/die Autor(en) 2020

Zusammenfassung Aus ihren theoretischen Konzeptionen und ihrer grundsätzlichen Haltung ergibt sich für die Integrative Gestalttherapie (IG) die Notwendigkeit, verschiedene diagnostische Perspektiven einzunehmen und zu integrieren. Die Vielfalt der Perspektiven ermöglicht es, der Absolutsetzung einer Perspektive entgegenzuwirken, und fördert die Einsicht, dass kein Diagnosesystem - sei es noch so ausgefeilt - die Wirklichkeit eines Menschen erfassen kann.

Menschen mit defizitären oder traumatisierenden Entwicklungsbedingungen (besonders in den ersten Lebensjahren) haben eingeschränkte Möglichkeiten zur Entwicklung ihrer strukturellen Fähigkeiten. Selbstregulierung, kreative Anpassung und Selbstaktualisierung in verschiedenen Lebensbereichen sind dadurch geprägt und die Lebensqualität oft deutlich beeinträchtigt.

Die Integration von Strukturdiagnostik in der IG-Diagnostik durch die Einbeziehung von OPD-2 ermöglicht eine differenziertere Einschätzung und Orientierung über Ressourcen und Defizite von Patient*innen.

Die mehrperspektivische diagnostische Herangehensweise - unter Einbeziehung der in der Leitlinie des Gesundheitsministeriums vorgesehenen Diagnoseschritte bei besonderer Berücksichtigung des unmittelbaren Beziehungsgeschehens und der Entwicklungsgeschichte - ermöglicht eine differenzierte Sicht auf die ganze Person im therapeutischen Kontext und im Lebensumfeld. Das unterstützt eine auf die Person und ihre Verarbeitungsfähigkeit abgestimmte thera-

\author{
M. Hochgerner \\ Messerschmidtgasse 40/4, 1180 Wien, Österreich \\ markus@hochgerner.net \\ P. Klampfl $(\bowtie)$ \\ Rudolfstraße 7/3, 3430 Tulln, Österreich \\ petra.klampfl@gmail.com
}

peutische Vorgehensweise, Schwerpunktsetzung und Beziehungsgestaltung, welche im therapeutischen Prozess dialogisch den jeweiligen Möglichkeiten und Bedürfnissen der Person angepasst werden kann. Menschen auf gutem Strukturniveau mit konflikthafter Thematik profitieren von anderen therapeutischen Angeboten als Personen mit strukturellen Defiziten. Im folgenden Beitrag soll die Verschränkung von Grundkonzepten der Integrativen Gestalttherapie und zentraler Punkte psychodynamischer Diagnostik (nach OPD-2) skizziert werden. Anhand einer kurzen Fallvignette werden aus diagnostischer und therapeutischer Perspektive und unter besonderer Berücksichtigung struktureller Einschätzung wesentliche therapeutische Vorgehensweisen dargestellt.

Schlüsselwörter Integrative Gestalttherapie · Diagnostische Perspektiven · Strukturdiagnostik · Prozess · Beziehung · Therapeutisches Vorgehen

Mrs. F. increasingly cares for herself

Processual structural diagnostics in Integrative Gestalt Therapy (IG)

Summary From its theoretical conceptions and its basic attitude, Integrative Gestalt Therapy (IG) is required to adopt and integrate different diagnostic perspectives. The diversity of perspectives makes it possible to counteract the absolute setting of one perspective and promotes the insight that no diagnostic system-no matter how sophisticated-can capture the reality of a person.

People with deficient or traumatising developmental conditions (especially in the first years of life) have limited opportunities to develop their structural abilities. Self-regulation, creative adjustment, and self-actualisation in various areas of life are characterised by 
this and the quality of life is often significantly impaired.

The integration of structural diagnostics in IG-Diagnostics by including OPD-2 allows a more differentiated assessment and orientation about resources and deficits of patients.

The multi-perspective diagnostic approach-including the diagnostic steps provided in the guideline of the Ministry of Health with special consideration of the immediate relationship and the history of development-enables a differentiated view of the whole person in the therapeutic context and in the living environment. This supports a therapeutic approach, setting of priorities and relational shaping that is tailored to the person and his or her ability to process, which can be adapted in the therapeutic process in dialogue to the respective possibilities and needs of the person. People at a good structural level with conflictual issues benefit from different therapeutic offers than people with structural deficits.

In the following article the interlocking of basic concepts of Integrative Gestalt therapy and central points of psychodynamic diagnostics (according to OPD-2) will be outlined. By means of a short case vignette, essential therapeutic procedures are presented from a diagnostic and therapeutic perspective and with special consideration of structural assessment.

Keywords Integrative Gestalt Therapy · Diagnostic perspectives · Structural diagnostics · Process . Relationship - Therapeutic approach

\section{Einleitung und Überblick}

Integrative Gestalttherapie als ein phänomenologisch-hermeneutisch begründetes Psychotherapieverfahren nahm in Zusammenhang mit kategorialer Diagnostik lange Zeit eine relativ kritische Position ein. Als Faktoren dafür können z. B. der phänomenologische und feldtheoretische Hintergrund aber auch die Betonung auf das unmittelbare Kontakt- und Beziehungsgeschehen genannt werden, was eine zuschreibend-objektivierende diagnostische Perspektive aus den Grundhaltungen heraus problematisch erscheinen ließ (Hochgerner et al. 2018, S. $179 \mathrm{ff}$.). Trotz einer kritischen Haltung gegenüber festschreibender Diagnostik wurde schon von den Gründerpersönlichkeiten auf die Notwendigkeit von diagnostischen Einschätzungen zur Orientierung und Interventionswahl hingewiesen (Perls et al. 2019, S. $310 \mathrm{f}$.).

Mit der Weiterentwicklung hin zu einem klinisch orientierten Verfahren wurden Erweiterungen und Differenzierungen diagnostischer Zugänge und Perspektiven im Verfahren notwendig und möglich, welche heute als mehrperspektivisches prozessuales und strukturbezogenes Vorgehen im Kontext der therapeutischen Beziehung etabliert sind.

Der ganzheitlichen Orientierung entsprechend wird der Mensch als untrennbare Einheit körperli- cher, seelischer und geistiger Aspekte in vielfältiger Verbundenheit mit seiner ökologischen und sozialen Umwelt verstanden (Petzold 2003, S. 408f.) wird somit vorrangig aus dem Erfassen der genannten Aspekte in ihrer Wechselwirkung und weniger der Analyse isolierter einzelner Phänomene gezogen. Gestalttheorie, Feldtheorie und beziehungstheoretische Ansätze sind ebenfalls konzeptionell bedeutsam. Dementsprechend werden Diagnostik und therapeutische Herangehensweise als phänomenologisches, feldbezogenes, ganzheitliches Vorgehen im Kontext unmittelbaren dialogischen Beziehungsgeschehens und als fortlaufender kontinuierlicher Prozess verstanden.

\section{Entwicklung des Selbst - Strukturperspektive}

Menschliche Entwicklung und Sein spielen sich von Anfang an in mitmenschlichen Beziehungen und in Auseinandersetzung mit der Umwelt ab und sind nicht davon zu trennen (Stern 2007, S. $47 \mathrm{ff}$.). Wachstum und Selbstentwicklung des Menschen erfolgen durch Integration unserer Erfahrungen im Kontakt mit der Umwelt und sind im Sinne eines differenzierenden Reifens als lebensbegleitender Veränderungsprozess zu verstehen. Strukturelle Fähigkeiten entwickeln sich im Beziehungsgeschehen mit wichtigen Bezugspersonen und prägen zeitlich überdauernd die zentralen Möglichkeiten des Selbst zu Differenzierung, Integration und Regulation im Umgang mit sich und anderen, also im Organismus/Umwelt-Feld (Rudolf 2013, S. $10 \mathrm{ff}$; Arbeitskreis OPD-2 2006, S. $114 \mathrm{f}$;; Hochgerner et al. 2018, S. 197 f.). Die Struktur einer erwachsenen Person kann demnach als Ergebnis eines Reifungsprozesses verstanden werden, für den zunehmende Differenzierung und Integration kennzeichnend ist, was die Möglichkeiten zur Selbst- und Beziehungsregulation, Selbstaktualisierung und kreative Anpassung an aktuelle Lebensumstände unterstützt. Strukturelle Entwicklung betrifft dabei zugleich das reifende Selbst und die sich differenzierenden Beziehungen.

Der Persönlichkeitsbegriff in der IG ist dynamisch und in die Lebenswelt eingebunden $\mathrm{zu}$ verstehen. Das Selbst ist leiblich zu verstehen und reicht von den ursprünglichsten biologischen Lebensprozessen als frühes, auftauchendes Selbst (Stern 2007, S. 61ff.) bis zu differenzierten Identitätskonstruktionen (Petzold 2003, S. $430 \mathrm{ff}$; Hochgerner et al. 2018, S. 191). Es entfaltet sich in Auseinandersetzung mit der Umwelt und entwickelt zur Orientierung in und zum „Kontakt“ mit der Umwelt einen ichhaft erlebten Prozess, den wir mit Hilfe immer weiter sich differenzierender Ich-Funktionen zur Selbstfürsorge und Selbstentwicklung nutzen (das „Ich“ wird dabei erlebbar als das Selbst in Aktion). Verstanden werden unter IchFunktionen vor allem Wahrnehmen, Fühlen, Denken, Wollen, Handeln, Erinnern und darauf aufbauende komplexe Ich-Fähigkeiten der Impulskontrolle, Nähe-Distanzregulation, Introspektion und Empathie. 
Im Laufe dieses Prozesses der dialogischen Selbstentwicklung werden zugleich durch Identifizierung und Identifikation mit Zuschreibungen durch die Umwelt Bilder von sich selbst (Selbst-Bilder) entwickelt, die in Summe die zeitlich überdauernden Merkmale der Identität bilden. Dieses Entwicklungsmodell macht es möglich, den Prozess, der mit Hilfe des Begriffs „Selbst“ beschrieben wird, als dynamische Strukturen bildend zu denken. Diese (leiblichen) Strukturen bilden die "Stütze“ für den im Vordergrund ablaufenden Erfahrungsprozess (Kontakt-Support). Im KontaktSupport-Konzept wird besonders auf die Wichtigkeit von Selbststützung als Voraussetzung für Kontakt und Entwicklung hingewiesen (L. Perls in: Sreckovic 2005, S. 53 ff.). Kontakt/Selbstentwicklung wird als Tätigkeit an der Grenze zwischen sich und dem Anderen verstanden, wobei Kontakt nur in dem Maß möglich ist, in dem Stütze dafür verfügbar ist.

\section{Entwicklungsstörungen}

Das Beziehungsgeschehen kann allerdings gestört sein und damit (besonders in den frühen Lebensphasen) den Entwicklungsprozess negativ beeinflussen, wobei die Förderung bzw. Störung einzelner Entwicklungsschritte in den verschiedenen Lebensphasen unterschiedlich gravierend ist. Maßgeblich sind die Varianz der Störbarkeit, also das Geflecht von belastenden und protektiven Faktoren in der sozialen Umwelt, aber auch die Passung zwischen dem Kind mit seiner genetischen Ausstattung (z.B. Vulnerabilität oder Resilienz) und den psychosozialen Belastungen in der Umwelt. Kommt es zu entsprechend massiven Störungen, die nicht ausreichend kompensiert werden können, bleiben die reifenden Funktionen vulnerabel oder defizitär und stehen dadurch für innere und interpersonelle Regulation nur eingeschränkt zur Verfügung. In Zusammenhang mit struktureller Problematik ist besonders das hohe Schädigungspotential von Bindungs- und Beziehungstraumatisierungen hervorzuheben und neurobiologische Modelle bestätigen, wie wichtig frühe dyadische Interaktions- und Regulationsprozesse zwischen Bindungspersonen und dem sich entwickelnden Kind in der Entwicklung der Fähigkeit zu Selbstregulation und Selbstberuhigung sind (Schore in Wöller 2013, S. $78 \mathrm{ff}$.). Markante Entwicklungsstörungen in den ersten Lebensjahren zeigen sich z.B. in Störungen der Stress- und Emotionsregulation, Bindung und im Hirnwachstum, in der Folge auch durch mangelnde Fähigkeiten zur Selbst- und Beziehungsregulation, aber auch Defizite in der Mentalisierungs- und Reflexionsfähigkeit (Wöller 2013, S. 136 ff.; Rudolf 2013, S. 20 f.).

Im Sinne eines Entwicklungsdefizits kann strukturelle Störung also bedeuten, dass bestimmte strukturelle Differenzierungen und Integrationsschritte nicht oder nur eingeschränkt erfolgt sind. Die Gefahr der Verkettung von Belastungsfaktoren in der weiteren Entwicklung steigt und die Bewältigungs- möglichkeiten für aktuelle Belastungen können nicht ausreichend entwickelt werden. Strukturelle Störungen zeigen sich dahingehend, dass PatientInnen über Jahre hinweg Schwierigkeiten haben, das eigene innere System und den Bereich der interpersonellen Beziehungen in Balance zu halten. Die Ganzheitlichkeit des Selbst und der reifen Identität mit seinen integrativen Funktionen wird gefährdet und in seiner Verfügbarkeit eingeschränkt, was sich anhand unterschiedlicher Integrationsniveaus psychischer Struktur erfassen und beschreiben lässt (siehe unten).

Grundsätzlich werden in der Integrativen Gestalttherapie verschiedene pathogen wirkende Einflussfaktoren im Entwicklungsverlauf erfasst. Betont wird dabei die Komplexität des Entwicklungsgeschehens hin zu Symptomatik und Krankheit im Sinne eines multifaktoriellen Geschehens mit jeweils individuellen bio-psycho-sozialen Faktoren. Krankheitsentwicklung wird in diesem Sinne sowohl aus einer beziehungsdynamischen Perspektive im aktuellen Organismus/ Umwelt-Feld als auch aus einer entwicklungsbezogenen strukturellen Perspektive im Lebensverlauf betrachtet (Petzold 2003, S. 447 ff.; Richter 2011, S. 60 f.; Rudolf 2013, S. $20 \mathrm{f}$.).

\section{Strukturbezogene Diagnostik und Vorgehensweise in der IG}

Rudolf (2013) formuliert in Zusammenhang mit struktureller Entwicklung und Diagnostik drei übergeordnete Zielrichtungen struktureller Funktionen: differenzieren, integrieren und regulieren. Diese strukturellen Fähigkeiten lassen sich anhand umschriebener Dimensionen diagnostisch einordnen und therapeutisch aufgreifen (Arbeitskreis OPD-2 2006, S. $259 \mathrm{ff}$;; Rudolf 2013, S. $14 \mathrm{ff}$.). Beispiele dafür sind die Differenzierung von eigenem Erleben und Verhalten (z.B. Gefühle, Unterscheidung zwischen innen und außen), die Integration von unterschiedlichen Beziehungserfahrungen und Selbstanteilen (z.B. positive innere Bilder von wichtigen Menschen oder Identität), aber auch die Regulation von eigenem Erleben und Verhalten (z. B. Impulsivität, Selbstwert oder Nähe-DistanzRegulation).

Im Unterschied zu einer rein deskriptiven Diagnostik ermöglicht eine psychodynamische Sichtweise ein tieferes Verständnis für die innere Dynamik und Gesamtsituation einer Person (Hochgerner 2005). In der multiaxial aufgebauten OPD-2 sind neben der deskriptiven ICD-10-Achse vier psychodynamische, am aktuellen Selbst- und Beziehungserleben orientierte Achsen (Krankheitserleben/Beziehung/ Konflikt/Struktur) eingerichtet (Arbeitskreis OPD-2 2006, S. 35). Im folgenden Fallbeispiel werden diese Achsen anhand konkreter Szenen dargestellt.

Im präsentierten Fall Frau F. und dem damit verbundenen strukturbezogenen psychotherapeutischen Vorgehen wird neben der klinischen Symptomatik das Strukturniveau der Patient*in und daraus abgeleitete 
therapeutisch hilfreiche Praxis in den Fokus gerückt: Therapeutischer Schwerpunkt, die Art der Beziehungsarbeit und Auswahl therapeutischer Methoden sind auf die strukturellen Defizite abgestimmt. Je nach Störungsgrad und Prozess variiert die therapeutische Position zwischen stützender (hinter), begleitender (neben) und konfrontierender (gegenüber) Haltung (Rudolf 2013, S. 127). Diese mehrperspektivische diagnostische Einschätzung im Beziehungskontext und besonders die damit verbundene Erfassung der strukturellen Fähigkeiten und Einschränkungen ermöglicht die Erarbeitung therapeutischer Schwerpunkte und Orientierung für therapeutische Vorgehensweise und Beziehungsgestaltung.

Wichtige gestalttherapeutische Konzepte sind z.B. das Kontakt-Support-Konzept, Verbesserung von Selbstprozessen, die Integration und Verbindung verschiedener Selbstanteile und Erlebensbereiche und die Verbesserung der Kontaktgrenze. Hilfreich sind z.B. die Arbeit an differenzierter Wahrnehmung und Bewusstheit und die Erweiterung funktionaler Möglichkeiten der Selbstregulation und Selbststützung, aber auch vielfältige Ansätze in Zusammenhang mit Kontakt- und Beziehungsgestaltung. Durch die Einbeziehung kreativer Medien und körperbezogener Interventionen können in diagnostisch begründeter Weise, abgestimmt auf das jeweilige Strukturniveau, die aktuelle Belastungsgrenze, den therapeutischen Prozess und die Zielvorstellung zur therapeutischen Verbesserung sowohl erlebnisorientierte und ausdrucksfördernde als auch übungszentriert-strukturierende Vorgehensweisen in mehr konfrontierender, begleitender oder stützender Weise einbezogen werden (Hochgerner et al. 2018, S. 200).

\section{Fallbeispiel}

\section{Erstkontakt}

Frau F., 24 Jahre, Technikerin mit Höherer Technischer Lehranstalt (HTL), wird an einer internistisch-psychosomatischen Abteilung mit dem BMI von 15,1 und in deutlich verwahrlostem Zustand (mangelnde Körperund Zahnhygiene, kein Wäschewechsel seit mehreren Wochen) zu einer achtwöchigen stationären Psychotherapie aufgenommen. Im medizinischen Vorstellungsgespräch an der psychosomatischen Ambulanz wird Frau F. als zurückhaltend, altklug und unsicher erlebt, jedoch durchaus geeignet, am fordernden achtwöchigen Therapieprogramm mit $110 \mathrm{~h}$ Psychotherapie in der geschlossenen Gruppe mit weiteren 5 Frauen und zwei Männern teilzunehmen. Die Erscheinung der jungen Frau wird auf der Station als eher auffällig wahrgenommen - sowohl die Kleidung als auch Frisur, Wortwahl und Beschreibung des Alltagsverhaltens entspricht mehr dem Lebensstil einer einsamen, alten Dame als einer jungen Erwachsenen.

Die Pflegekräfte beschreiben Frau F. als im Erstkontakt oberflächlich freundlich, angepasst, jedoch auf der Station mit Sonderwünschen insistierend bezüglich Ausgangsregelung, dem Essen, das sie nicht zeitgleich mit den Anderen einnehmen möchte und dem Wunsch, nur alle zwei Tage Essen zu bestellen, da sie „ohnehin kaum was isst - was für eine Verschwendung!" Sie sitzt meist auf ihrem Bett, liest und nimmt keinen Kontakt zu den gleichaltrigen Mitpatient*innen auf. Sie erkundigt sich nach psychologischer Betreuung und möchte mit der klinischen Psychologin bereits im Erstgespräch vor Beginn des Aufenthaltes weitere Rehamaßnahmen für danach planen („Ein Aufenthalt wird da bei mir wohl nicht reichen, oder?"). Nach der internistischen Abklärung ihres gesundheitlichen Status und der vorliegenden Therapiemotivation wird Frau F. in den achtwöchigen stationären Aufenthalt zur Psychotherapie aufgenommen.

\section{Therapeutisch-diagnostische Reflektion 1 - Diagnostische Hinweise aus Berichten und Befunden}

Die erhöhte Komplexität der Befunderhebung im Kontext/Behandlungsfeld Spital bietet förderliche und hemmende Momente: Eine Vielzahl von Interaktionen und Daten (medizinisches Gespräch, Pflegegespräch, akute Befunderhebung und Krankengeschichte) ermöglichen ein komplexes Bild der Person mit leidvoller Symptomatik. Zugleich ist mit einer Präformierung der Wahrnehmung zu rechnen: Die Institution neigt zur (vor-)schnellen Kategorisierung von „Patienten*innengut“ als „interpersonale Abwehr" (Mentzos 1988, S. $21 \mathrm{ff}$., S. $99 \mathrm{ff}$.) sowohl aus den Blickwinkeln der einzelnen Gesundheitsberufe Medizin, Pflege, Klinische Psychologie als auch im Sinn einer Reduktion komplexer Wahrnehmung und Angst-/Stressminderung der Behandler*innen zu „institutionalisierten Abwehr" (Mentzos ebendort), die zur einfachen Kategorienbildung anhand der Linie „angenehmer vs. unangenehmer Patient“ neigt. Zugleich sind auch erste diagnostische Einschätzungen möglich: Die gegenwärtige Schwere der Symptomatik (OPD 2 Achse 1) ist mit hoch (Stufe 3 von 4 Schweregraden) mit deutlichen körperlichen und seelischen Symptomen anzusetzen. Der Wert auf der GAF-Skala (Global Assessment and Functioning Scale, DSM IV) beschreibt das psychische, soziale und berufliche Funktionsniveau, das hier auf einer Skala von 100 (optimale Funktion in allen Bereichen) bis 1 (ständige Lebensgefahr und anhaltende Unfähigkeit zur Lebensgestaltung) mit 45 eingestuft werden kann (ernsthafte/starke Beeinträchtigung in mehreren Bereichen OPD-2 2006, S. $149 \mathrm{ff}$.).

In der freien Praxis wäre hier im Erstkontakt auf die initiale Kontaktaufnahme (Überweisungskontext, erste Worte am Telefon, erste Begegnung in der Praxis) $\mathrm{zu}$ achten. Dieser „erste Blick“ wird hier durch „erste Information“ im Vorlauf des Behandlungsangebotes „Psychotherapie“ abgelöst bzw. ergänzt. Die 
geforderte Einschätzung der Krisenhaftigkeit wurde bereits geleistet, die Frage einer möglichen hilfreichen psychotherapeutischen Beziehung im ärztlichen Gespräch erhoben und damit wesentliche Punkte der psychotherapeutischen Diagnosestellung (Diagnostik-Leitlinie des Bundesministeriums für Gesundheit 2004, S. $10 \mathrm{f}$; im Weiteren „Leitlinie“ genannt) erfasst. Offen bleibt die Überprüfung und Bestätigung/ Relativierung der ersten getroffenen Einschätzung und Klärung der „Symptomatik in Relation zur Persönlichkeit“ im ersten psychotherapeutischen Kontakt, um eine letztendliche Indikation zur psychotherapeutischen Behandlung stellen zu können: Dabei ist die ICD-10 Diagnostik mit Anorexia Nervosa (F 50.0) eindeutig erhebbar.

Der gestaltherapeutische Zugang bietet hier verfahrensspezifischen Zugang in der Erfassung des Kontaktverhaltens bzw. Kontaktunterbrechungen, die sich sowohl auf das Erleben der Patient*in mit sich selbst als auch Ihrer Umgebung beziehen können. Dieses Kontaktverhalten erzeugt sowohl emotionale Resonanz in der Therapeut*in (einfühlend und als Gegenübertragungsreaktion) als auch eine therapeutische „Szene“, in der wesentliche Merkmale der PersonUmweltrelation erfassbar werden. Dieses Moment des „mit sich und anderen in Beziehung sein“ bildet sich hilfreich auch in OPD 2 Achse 2 ab (siehe unten, Reflexion 2).

Im folgenden psychotherapeutischen Erstkontakt erscheint Frau F. geordnet, konzentriert und berichtet emotional scheinbar unbeteiligt und sachlich, sie sei gegen das Abraten der Familie an der Abteilung vorstellig geworden, da ihre Mutter meinte, sie habe wohl kein Recht zur Aufnahme, wenn sie noch nicht sondenpflichtig (ab BMI 14,0) künstlich mit Nasensonde ernährt werden müsse und damit einer krankeren Person einen Therapieplatz wegnehmen könnte. Beide Eltern bezweifeln die Krankheitswertigkeit des Essverhaltens und diskutieren nach der Veröffentlichung ihres Behandlungswunsches die Frage, ob eventuell die Zusammensetzung der Nahrung Schuld am „Unglück der Tochter“ (Zitat Mutter) haben könnte. Ihr Vater schlägt zur Gesundung ein technisches Studium vor, denn „jemand ohne Doktorat bis zum 26. Lebensjahr ist ohnehin kein lebenswerter Mensch“. Sie selbst habe jedoch „allmählich Bedenken“ wegen ihres Essverhaltens.

\section{Kommentar}

OPD-2 Achse I (Krankheitserleben und Behandlungsvoraussetzungen) erfasst vor allem Beschwerdesymptomatik und Behandlungserwartungen. Der Akzent liegt hier weniger auf dem Krankheitsverhalten als auf Erlebenselementen, Motivationen und vorhandenen Ressourcen. Bedeutsam ist das mehrperspektivische Erfassen des Phänomens (z. B. wie zeigt sich die Belastung und wie wirkt sich die Symptomatik auf die
Lebensrealität der Person aus). Fokussiert wird das eigene Erleben der Person.

Therapeutisch-diagnostische Reflektion 2 atmosphärisch-szenische und Beziehungsdiagnostik

Die Diskrepanz der erzählten inadäquaten und unempathischen familiären Reaktion bei gleichzeitig gezeigter, leicht ironisierender Unbeteiligtheit im Erstgespräch löst in der Therapeut*in dreierlei aus: Ungläubiges Staunen begleitet von Empörung zur Resonanz in der Familie, eine als erhöht spürbare Anstrengung, durch besonders kluges und umfassendes Explorieren die Patient*in zu erreichen und ein ganz konträr-unpassendes Gefühl der Langeweile und Gereiztheit und beginnender Unkonzentriertheit, wenn Frau F. mit einer manieriert-künstlichen Sprache ihr Lebensfeld beschreibt („Ach ja, by the way, was ich noch zu meinen wirklich lustigen Großeltern erzählen wollte ..."). In der Therapeut*in taucht spontan das Bild eines dressierten traurigen Pudels, auf einem Seidenpolster sitzend, auf. Die geschilderten Szenen, das damit verbundene Erleben und der diffuse Affektausdruck gepaart mit differenzierter Beschreibung der handelnden Personen sind hoch inkongruent und unpassend.

Die Patientin erscheint klischeehaft eingeengt, überkontrolliert und versucht alle realen und vermuteten „Aufgaben“ im Erstgespräch zu erfüllen, bleibt als Person in der mangelnden Verbindung von Affekt, Szene und Mitteilung jedoch klischeehaft unzugänglich. Das Kontaktverhalten erscheint im gestalttherapeutischen Sinne retroflexiv - alle Affekte werden nach innen und im Zweifelsfall gegen sich selbst gerichtet - im Sinne der OPD 2 im passiven Modus als Selbstunsicherheit erlebt und als „stiller Schrei“ und Apell zur Hilfe von außen szenisch gestaltet.

Beziehungsdiagnostisch (OPD Achse II, Beziehung) zeigt sich, dass die Patient*in sich sehr anstrengt zu genügen und beachtet zu werden, dies jedoch sowohl innerhalb der Familie als auch unter Freund*innen enttäuschend oft kaum gelingt. Beziehungsdynamisch formuliert (OPD-2 2006, S. 197) hat Frau F. immer wieder den Eindruck zu langweilen, überhört und missverstanden $\mathrm{zu}$ werden. Frau F. erlebt manche Personen (Lehrer, Schulkolleg*innen, Kolleg*innen einer kirchlichen Jugendorganisation) ihr gegenüber mit zuerst Überengagement („das ich ja gar nicht verstehe oder verdiene!"), dass dann meist bald enttäuschend in Desinteresse und Ablehnung umschlägt. Eine ähnliche Reaktion entstand in der Therapeut*in schon in den ersten Kontaktmomenten: Mitgefühl und Überengagement einerseits und aufkeimendes Desinteresse und eine Szene der Fremdheit und Unbezogenheit im Bild des Pudels anderseits. Dasselbe Muster wurde im Behandler*innenteam der Pflege im Spitalsaufenthalt erzeugt: Detaillierte Berichte im Team zu Frau F. wechselten mit Pflegekräften, die 
nicht einmal bemerkt hatten, ob Frau F. auf der Station gewesen war oder den Namen der Patient*in bis zum Schluss nicht richtig zuordneten.

\section{Kommentar}

OPD-2 Achse II (Beziehung) erhebt das grundsätzliche Beziehungsverhalten der Person und greift damit zentrale Themen von Kontakt- und Interaktionsgeschehen einerseits und Aspekten der Beziehungsgestaltung andererseits auf. Erfasst werden Schilderungen von relevanten Interaktionen mit bedeutsamen anderen Menschen in Form von Beziehungsepisoden. Die Beziehungsdiagnostik fokussiert dabei sowohl auf die Erlebensperspektive der Person (Patient*in) als auch auf die Erlebensperspektive von anderen (auch die Therapeut*innenperspektive) innerhalb und außerhalb der Therapiesituation. Bedeutsam ist das Geschehen im Hier und Jetzt ebenso wie die Beschreibung des Beziehungs-Erlebens im Außen.

\section{Erste Gruppentherapie}

Frau F. berichtet, dass sie sich in ihrer finanziell wohlhabenden Familie an kein einziges (!) gemeinsames Essen in ihrer Lebensgeschichte erinnern könne: Alle nehmen sich etwas aus dem Kühlschrank, „der jedoch ohnehin meist leer ist“. Es werde jedoch viel über gesundes Essen gesprochen. Sie horte mit ihrer Schwester im gemeinsamen Zimmer meist eine Plastiktasche mit „gesunden und billigen“ Lebensmitteln. Frau F. dissimuliert die Krankheitswertigkeit ihres Zustandsbildes und imponiert in der Psychotherapiegruppe durch hohes, unempathisches Engagement bezüglich der Mitpatient*innen durch Tipps, Diskussionsangebote und ausufernde, kluge Argumente „wie man das auch noch anders sehen könnte“.

\section{Kommentar}

\section{Selbst- und Beziehungsregulation/Körpererleben}

Einschränkungen der Selbstregulation zeigen sich häufig dadurch, dass in der Entwicklung beruhigende und fürsorgliche Bezugspersonen nicht ausreichend zur Verfügung gestanden sind und positive Beziehungsmuster nicht verinnerlicht werden konnten (siehe unten). Im Entwicklungsverlauf dominieren Defizite, Traumatisierungen oder inkonsistentes und unberechenbares Verhalten der Bezugspersonen. Unzureichend ausgebildete Ich-Funktionen und Selbstgrenzen führen zu einer Beeinträchtigung der Selbstregulation und der psychosozialen Anpassung mit vielfältigen Störungen. Deutliche Probleme zeigen sich z. B. im Bereich der Emotionsregulierung (Frau F. erkennt nicht, wann ihr Beitrag in der Gruppe angenommen werden kann, sie verwendet im Sinne der psychischen Selbstregulation intellektualisierende und rationalisierende Momente der Abwehr), der Mentalisierungsfähigkeit (spricht ungerührt weiter auch wenn die angesprochene Mitpatientin bereits Tränen unterdrückt) und Ich-Integration (Wöller 2013, S. $99 \mathrm{ff}$.).

Einschränkungen in der Kontakt- und Beziehungsregulation zeigen sich in vielerlei Art sowohl im unmittelbaren therapeutischen Beziehungsgeschehen als auch in Schilderungen der Patient*innen aus ihrem Lebensumfeld. Menschen mit strukturellen Störungen verlieren unter Belastung in gewisser Weise ihre Selbstwirksamkeit: Sie sind überflutet von Verzweiflung, wütender Erregung und Panik oder erleben sich leer und erstarrt. In dieser Verfassung können sie auch keine hilfreichen und beruhigenden Beziehungen herstellen. „Beziehungsmuster, die in der Außenperspektive als dysfunktional erscheinen, [sind] primär adaptive Regulierungsleistungen im zwischenmenschlichen Bereich, mit denen Patienten eine Bewältigung sowohl ihrer Konfliktneigungen als auch ihrer strukturellen Vulnerabilitäten versuchen“ (OPD-2 2006, S. 137): Frau F. beschreibt ein Jahr später in der ambulanten Nachsorgegruppe, wie sehr sie emotionale Momente in der Gruppe (siehe oben) mit Ratlosigkeit und aufkeimender Panik erfüllten. Oft versuchte sie sich bewusst anzuspannen, um sich „irgendwie“ selbst zu spüren und wäre sie gerne aus der Gruppe geflüchtet.

Körpererleben und Körperbezug sind stark durch strukturelle Defizite und Folgen von Traumatisierungen geprägt. Der Körper wird häufig als Quelle unlustvoller Spannungszustände erlebt und das Körpererleben abgespalten. In der angeführten Fallsituation (siehe Gruppenszene oben) zeigen sich Retroflexion, Anästhesierung und Spaltung als drei nachhaltig wirkende Abwehr- und Bewältigungsstrategien (Petzold 2003, S. 585 ff.), die Menschen aufgrund massiver Defizite oder Überstimulierungen in ihrer Entwicklung kompensatorisch etablieren können und die sich meist als diffuse Stimmungen, somatische Sensationen oder Atmosphären deutlich machen, welche sich sprachlich jedoch vorerst kaum ausdrücken und bearbeiten lassen.

Therapeutisch-diagnostische Reflektion 3 Erfassung der inneren und umgebenden Lebenswelt

Frau F. wird nach erfolgter Eingewöhnung auf der Therapiestation als deutlich jünger wirkende, mädchenhafte Erscheinung sichtbar, die sowohl in ihrer körperlichen Erscheinung und im Sozialverhalten eher 16-jährig als Mitte 20 erscheint. Die sozialen Bezüge sind überwiegend in der Ursprungsfamilie. Soziale und kulturelle Interessen finden hochritualisiert in einer Jugendorganisation am Wochenende und einmal wöchentlich statt. Kurze, wechselnde und unterschwellig sexualisierte Kontakte in der Gruppe führen zu meist heimlichen Affären mit keiner adäquaten Beziehungskonstanz. Der Versuch, möglichen Partnern unbedingt zu gefallen und deren Ansprüchen zu genügen, führt gefühlt zur Auflösung ihres Selbstgefühls 
und zu keinem dialogischen Gegenüber in einer Partnerschaft. Das Geld, das sie in einer Halbtagsstellung in einem Planungsbüro verdient, bleibt ungenutzt. Sie lebt weiter in einer Wohnung der Eltern im selben Haus.

Hinweise zur Einschätzung der strukturellen Stabilität/Fragilität (OPD Achse IV) in Zusammenhang mit ICD-10 und IG-spezifischen Kategorisierungen zeigen eine gute Fähigkeit zur Selbstreflexion, jedoch eine deutliche Einschränkung und mäßige Integration im Bereich Affektdifferenzierung und Identität: Vermeidendes Verhalten in der eingeschränkten Gefühlswahrnehmung (z.B. negative Affekte) ergänzen sich mit Einbrüchen im Selbstbild (z. B. bei erhöhter innerpsychischer Belastung). Die Fähigkeit zur Abgrenzung (z.B. unsichere Selbst- und Objektabgrenzung) ist deutlich reduziert und führt zu Rückzug im Sozialverhalten. Die Erfassung dieses Verhaltens wird in der IG als dysfunktionale Anpassungsleistung zur Stabilisierung des Selbst erfasst. Eine Codierung unter Soziale Phobie F. 40.1 (ICD 10) als Co-Morbidität wird im Team erwogen, jedoch im Laufe des Aufenthaltes verworfen. Vielmehr bietet sich im weiteren Verlauf das Bild einer lavierten, atypischen Depression F32.8 (ICD 10), die mehr den Zustand der Verwahrlosung und Achtlosigkeit in der körperlichen Versorgung erfasst: Frau F. „vergisst“, sich zu Waschen, zu Essen, erinnert sich nicht an die letzte Nahrungsaufnahme, hat kein Hungergefühl. Ebenso ist das Körpererleben bis zur Fühllosigkeit deutlich eingeschränkt (OPD 2 Achse IV, Item Körperselbst) und die Kommunikation nach außen (ebendort, Item Kontaktaufnahme) gering integriert: Zurückgezogenheit kann jederzeit in distanzloses Kontaktverhalten umschlagen.

\section{Kommentar}

\section{OPD-2 Achse III und IV}

Achse III (Konflikt) untersucht die Bedeutung innerer Konflikte der Person und ihre Bewältigungsformen. Unterschieden werden sieben Konfliktkonstellationen mit jeweiliger Differenzierung in „aktiven Modus“ und „passiven Modus“ (Arbeitskreis OPD-2 2006, S. $206 \mathrm{ff}$.). Konfliktthemen sind z. B. Individuation versus Abhängigkeit, Unterwerfung versus Kontrolle oder Versorgung versus Autarkie.

Zur Erfassung überdauernder Konflikte werden im diagnostischen Gespräch verschiedene Lebensbereiche - vergleichbar dem Konzept der 5 Säulen der Identität in den angeführten Lebensdimensionen Leiblichkeit, soziales Netz, Arbeit, materielle Situation und Werte (Petzold in Richter 2011, S. 55) - angesprochen und die sich wiederholenden konflikthaften Themen im Lebenskontext und Lebenskontinuum erhoben. Neben der Schilderung der Person spielen auch - sehr nah am gestalttherapeutischen Vorgehen - die Erfassung eines Leitaffektes, die szenische Information im unmittelbaren Geschehen und die
Gegenübertragung eine wesentliche Rolle. Die Achsen „Konflikt“ und „Struktur“ verhalten sich wie Figur zu Grund, da das Vorhandensein zeitüberdauernder, unbewusster Konflikte an bestimmte strukturelle Voraussetzungen geknüpft ist.

Achse IV (Struktur) bildet die Fähigkeiten und Defizite psychischer Strukturen ab. Struktur bezieht sich auf die „Verfügbarkeit über psychische Funktionen in der Regulierung des Selbst und seiner Beziehung zu den inneren und äußeren Objekten" (Arbeitskreis OPD-2 2006, S. 255). Strukturelle Störungen sind durch unzureichende Verfügbarkeit dieser Funktionen oder ihre Vulnerabilität gekennzeichnet, wobei sich die strukturellen Muster im interaktionellen Handeln, in der direkten Begegnungssituation, in Schilderungen der Personen über ihren Alltag und ihrer Lebensgeschichte zeigen. Selbst- und Beziehungsregulation, wie auch Reflexion, sind dadurch nur eingeschränkt möglich (siehe Gruppenbeispiel oben).

Die auf der Strukturachse erfassten strukturellen Funktionen werden in vier Dimensionen gegliedert, welche sich jeweils auf das Selbst und die Anderen beziehen lassen (Arbeitskreis OPD-2 2006):

- Selbstwahrnehmung und Objektwahrnehmung: Fähigkeit, sich selbstreflexiv wahrzunehmen bzw. andere ganzheitlich und realistisch wahrzunehmen

- Steuerung des Selbst und der Beziehungen: Fähigkeit, eigene Impulse, Affekte und den Selbstwert zu regulieren bzw. den Bezug zum anderen regulieren zu können

- Emotionale Kommunikation nach innen und außen: Fähigkeit zur inneren Kommunikation (Erfassung und Umgang mit Affekten und Fantasien) bzw. zur Kommunikation mit anderen

- Innere Bindung und äußere Beziehung: Fähigkeit, gute innere Bilder zur Selbstregulierung zu nutzen bzw. sich in realen Beziehungen zu binden oder auch wieder zu lösen

Die Gesamtheit der gut verfügbaren bis nicht zur Verfügung stehenden strukturellen Fähigkeiten kennzeichnet das Funktionsniveau der Struktur. Es werden vier Niveaus der strukturellen Integration unterschieden: gut integriert/mäßig integriert/gering integriert oder desintegriert (Rudolf 2013, S. 54 ff.; Arbeitskreis OPD-2 2006, S. 255 ff.).

\section{Zweite Einzeltherapie}

Der Therapeut schlägt vor, auf einem Tisch mit Hilfe von Gegenständen (Steine, kleine Figuren aus verschiedenen Materialien, Holzwürfel etc.) die Familie und die jeweilige emotionale Bezogenheit der Familienmitglieder durch unterschiedliche Abstände in den einzelnen Positionierungen darzustellen. Akribisch werden die nahen Angehörigen und auch entfernte Verwandte ausgesucht und positioniert. Als die Gestaltung aus Sicht der Patientin abgeschlossen ist weist der Therapeut darauf hin, dass die Patientin 
selbst in der Gestaltung nicht vorkomme. Frau F. nimmt dies scheinbar ungerührt zur Kenntnis: „Ach ja stimmt, wie immer, mich haben auch alle immer vergessen ... “. Der Hinweis des Therapeuten, sie habe ja sich selbst in dieser Gestaltung nicht bedacht, wird mit Vorbehalt („Ist das eventuell ein Zufall? Wie war der Gestaltungsvorschlag formuliert?“) zur Kenntnis genommen. Nur zögerlich wählt Frau F. ein kleines Wollschaf als Symbol für sich und stellt es vorsichtig an den Rand der Gestaltung mit Blickrichtung hin zu sich selbst. Dieses Wollschaf wird im Laufe der Stunden Sinnbild der Bedürftigkeit und „weichen“ Gefühle, die Frau F. als missachtetes Kind und gefühlt „Schwarzes Schaf der Familie“ nicht haben durfte. Spontan entschließt sich die Gruppe, gemeinsam T-Shirts mit schwarzen Schafen anzuschaffen und damit einen Raum der Solidarität mit den ungesehenen Teilen der eigenen Bedürfnisse zu schaffen.

\section{Therapeutisch-diagnostische Reflektion 4 - Diagnostische Gesamtschau: therapeutische Haltung, Fokus und Vorgehen}

Frau F. wurde in der Folge mit der Diagnose nach ICD 10/F 50.0 (anorexia nervosa) und im Sinne einer Co-Morbidität bezüglich F32.8 (atypische Depression) behandelt. Nach Achse IV OPD 2 (Struktur) konnte Frau F. als durchgehend mäßig integrierte Persönlichkeit mit besonderer Fragilität (mäßig/ gering integriert) im Bereich Selbstwert (Hohe Unsicherheit, durch Leistung gestütztes Selbsterleben) und im Bereich Bindung (geringe Fähigkeit zur Aufrechterhaltung naher Beziehungen und Schutz naher Beziehungen, dem Unvermögen für sich genügend zu sorgen und der mangelnden Fähigkeit, in chronischen Notlagen die Hilfe anderer zu aktivieren) erfasst werden.

Auf Achse III OPD 2 (Konflikte) ist eine ausgeprägte Verunsicherung im Bereich Identität und Autarkie festzustellen: Frau F. kann lange Zeit keine ihr genügend passende Selbst- und Weltsicht beschreiben, die nicht sofort wieder in existenziellem Zweifel an sich selbst aufgelöst wird. Selbstversorgung durch Essen und angemessene Körperhygiene ist kaum möglich. Das therapeutische Vorgehen orientiert sich an der Stärkung des Selbstwerts durch maßvolle empathische Interventionen, Ermutigung $\mathrm{zu}$ neuem, experimentellem Verhalten und vermehrte Abgrenzung (OPD: Regulationsfähigkeit vermehren) gegenüber der pathologischen Interaktion in der Ursprungsfamilie in einer therapeutischen Position „neben der Patient*in“ (Rudolf 2019, S. 92f.), in der „eine Außenbeobachtung, die zugleich behutsam zur Introspektion einlädt" möglich wird (Rudolf, ebendort; OPD: Differenzierungsfähigkeit fördern). Die therapeutische Haltung ist ermutigend-explorierend und in Momenten der strukturellen Überlastung stützend und entlastend. Dies ermöglicht der Patient*in, sowohl den Eindruck von genügender Kontrolle des therapeutischen Prozesses zu haben (Förderung der Autarkie) und sich nicht einer therapeutischen „Expert*innenschaft“ unterwerfen zu müssen als auch Ermutigung $\mathrm{zu}$ eigengesteuertem Verhalten $\mathrm{zu}$ erfahren und damit hilfreiche Versorgung zuzulassen. Der immer wieder geforderte und geförderte Affektabgleich zwischen innerem Erleben/innerer Haltung, äußerer Haltung und Verhalten und dabei eingebrachter bestätigender maßvoller empathischer Resonanz in der therapeutischen Beziehung verbindet ein sich entwickelndes Selbstbewusstsein mit wohlwollendempathischer Sicherung des Erlebens und Verhaltens durch die Therapeut*in (OPD: Internalisierung guter Beziehungserfahrungen ermöglichen).

Als expliziter (mit der Patient*in geteilter) Fokus wurde vermehrte Selbstfürsorge im Bereich tägliche körperliche Pflege/Essen und Vermehrung der Sozialkontakte außerhalb der Familie vereinbart. Der implizite (die therapeutische Haltung und Vorgangsweise steuernde) Fokus formierte sich im begleitenden Modus (siehe oben) um die Aufmerksamkeit bezüglich vermehrter Differenzierung der eigenen Affekte durch spiegelnde Unterstützung in geteilter Aufmerksamkeit (shared awareness, Sander 1983): Diese Erfahrung eines „geteilten Gewahrseins, daß nämlich Ausdruck oder Mitteilung des eigenen inneren Erlebens von einer anderen [„Person“, Anmerk. d. Verf.] wahrgenommen, geteilt und verstanden werden, verleiht diesem Erleben Realität" (Grothe 1991, S. 27 f.). Die darauf aufbauende Unterstützung experimentellen, explorativen Verhaltens fördert die flexiblere Gestaltung altersgemäßer weiblicher Identität.

Eine erste anhaltende Verbesserung der strukturellen Verfasstheit wurde von der Patient*in aus sozial angespannten Situationen berichtet, als sie in der jeweiligen Situation vorhergehende therapeutische Sequenzen und Sätze abrufen konnte, die ihr halfen, die zu bewältigende Situation hilfreich zu gestalten. Dadurch konnte sich ein vermehrtes Gefühl der Selbstsicherheit und eine Ermutigung zur Beachtung eigener Impulse etablieren.

Zwei Jahre nach Beendigung des stationären Aufenthaltes, in einer ambulanten Nachsorgegruppe beschreibt Frau F.: „Das war damals im Erstgespräch das erste Mal im Leben, dass ich bemerkt habe, dass jemand an mich denkt und für mich sorgt ... und ich auch nicht gelernt hatte an mich zu denken“.

\section{Kommentar}

Reflexionsfähigkeit und Abwehrvorgänge sind durch strukturelle Defizite häufig deutlich eingeschränkt geprägt. Menschen mit strukturellen Störungen haben Schwierigkeiten, sich selbst zum Gegenstand der Reflexion $\mathrm{zu}$ machen und sich differenziert selbst $\mathrm{zu}$ begegnen. Aus der Strukturperspektive ist die Entwicklung und Beschaffenheit eines seelischen Binnenraums im Sinne „einer inneren Bühne“ (Rudolf et al. 2008, S. 20) bedeutsam. Wesentliche Aspekte da- 
bei sind z. B. die Fähigkeit, Konflikte innerpsychisch abzuwägen und auszutragen (als regulative Funktion), aber auch die Fähigkeit, das eigene innere Geschehen auf dieser „inneren Bühne“ wahrzunehmen (als reflexive Funktion), aber auch Beziehungserfahrungen zu verinnerlichen (Klampfl 2018, S. 48). Über die Einbeziehung von kreativen Medien kann die Arbeit an und mit dieser „inneren Bühne“ über die Symbolisierung unterstützt und Reflexionsprozesse angeregt werden (Hochgerner 2015, S. 108f.).

Unter Abwehr wird ein dynamischer Vorgang verstanden, der das Bewusstsein sowohl vor überfordernden inneren Reizen als auch vor inneren Auswirkungen durch äußere, überfordernde Reize schützt (siehe Fallbeispiel oben, Gruppenstunde). Für die Einschätzung des Strukturniveaus relevant ist die Art der Abwehrorganisation. Bedeutsam ist dabei z. B. der qualitative Sprung zwischen mäßig und gering integriertem Strukturniveau. Während die Abwehr auf mäßig integriertem Strukturniveau noch weitgehend durch intrapsychische Vorgänge gekennzeichnet ist, widerspiegelt sich Abwehr auf gering integriertem Strukturniveau bereits deutlich auf interpersoneller Ebene.: Problematiken werden im Außen gestaltet/in die Beziehungen hineingetragen und führen sekundär wieder zur Verinnerlichung negativer Selbst- und Beziehungserfahrungen.

Während Menschen mit neurotisch-konflikthafter Problematik (OPD: Gut strukturiert) vor allem unter blockierter Eigenaktivität und damit verbundenen pathogenen Überzeugungen (z. B. negativer Selbstwert, Erwartung von Misserfolg und Zurückweisung) und dysfunktionalen Verhaltensbereitschaften (z. B. Rückzug und Unterordnung) leiden und im emotionalen Erleben Zurückhaltung und negative Affekte vorherrschen, sind es bei struktureller Störung (OPD mäßig/ gering/desintegriert) viel mehr das Verhalten anderer Menschen, das schwer zu ertragen ist, eine grundsätzlich eingeschränkte Verfügbarkeit der Fähigkeit zur Regulation des Selbst und seiner Beziehungen und im Speziellen die eingeschränkte Fähigkeit des Selbstverständnisses und der Affektregulierung (Rudolf 2013, S. $51 \mathrm{ff}$.). Eine strukturelle Störung „ist keine umschriebene klinische Einheit" (Wöller und Kruse 2015, S. 311), das Erscheinungsbild ist vielmehr durch das Ausmaß der strukturellen Vulnerabilität und durch den Grad der strukturellen Desintegration gekennzeichnet. Entscheidend ist die aus der Einschätzung der strukturellen Gegebenheit entwickelte hilfreiche therapeutische Haltung und eine patientengemäße Formulierung des therapeutischen Fokus und Ziels.

Interessenkonflikt M. Hochgerner und P. Klampfl geben an, dass kein Interessenkonflikt besteht.

Open Access Dieser Artikel wird unter der Creative Commons Namensnennung 4.0 International Lizenz veröffentlicht, welche die Nutzung, Vervielfältigung, Bearbeitung, Verbreitung und Wiedergabe in jeglichem Medium und Format erlaubt, sofern Sie den/die ursprünglichen Autor(en) und die Quelle ordnungsgemäß nennen, einen Link zur Creative Commons Lizenz beifügen und angeben, ob Änderungen vorgenommen wurden.

Die in diesem Artikel enthaltenen Bilder und sonstiges Drittmaterial unterliegen ebenfalls der genannten Creative Commons Lizenz, sofern sich aus der Abbildungslegende nichts anderes ergibt. Sofern das betreffende Material nicht unter der genannten Creative Commons Lizenz steht und die betreffende Handlung nicht nach gesetzlichen Vorschriften erlaubt ist, ist für die oben aufgeführten Weiterverwendungen des Materials die Einwilligung des jeweiligen Rechteinhabers einzuholen.

Weitere Details zur Lizenz entnehmen Sie bitte der Lizenzinformation auf http://creativecommons.org/licenses/by/4. 0/deed.de.

\section{Literatur}

Arbeitskreis, O. P. D. (2006). Operationalisierte Psychodynamische Diagnostik OPD-2. Das Manual für Diagnostik und Therapieplanung. Bern: Huber.

Diagnostik-Leitlinie des Bundesministeriums für Gesundheit (2004) Diagnostik-Leitlinie für Psychotherapeutinnen und Psychotherapeuten. Begriffserklärungen und Leitlinien zur psychotherapeutischen Diagnostik. https://www.sozialministerium.at/dam/jcr:7d05660cb825-4764-90ac-18d0b20d012d/Diagnostik-Leitlinie, 28.04.2020,_barrierefrei_(BMSGPK).pdf. Zugegriffen: 8.6.2020

Grothe, K. D. (1991). Weder Autismus noch Symbiose. Neuere Erkenntnisse der psychoanalytischen Entwicklungspsychologie aus der Sicht der Kleinkindforschung. In: Konzentrative Bewegungstherapie. Zeitschrift des DAKBT, 20, 19-32.

Hochgerner, M. (2005). Psychodynamische Diagnostik in Psychotherapie und Beratung. Masterthese. Krems: Donau UniversitätKrems, Zentrum für Psychosoziale Medizin.

Hochgerner, M. (2015). Die Verwendung von Gegenständen in der Psychotherapie. Theorie und Praxis des heilsamen Gebrauchs konkreter Objekte in der psychotherapeutischen Behandlung Erwachsener. Saarbrücken: Akademikerverlag.

Hochgerner, M., Klampfl, P., \& Nausner, L. (2018). Diagnostische Perspektiven der Integrativen Gestalttherapie. In M. Hochgerner, H. Hoffmann, L. Nausner \& E. Wildberger (Hrsg.), Gestalttherapie(S. 179-205). Wien: Facultas.

Klampfl, P. (2018). Musiktherapeutische Arbeit mit strukturellen Fähigkeiten und Defiziten von PatientInnen. Zur Erfassung struktureller Funktionen im musiktherapeutischen Kontext. Diplomarbeit. Wien: Institut für Musiktherapie. Universität für Musikund darstellende Kunst.

Mentzos, S. (1988). Interpersonelle und institutionalisierte Abwehr. Frankfurt: Fischer.

Perls, F., Hefferline, R. F., \& Goodman, P. (2019). Gestalttherapie. Grundlagen der Lebensfreude und Persönlichkeitsentfaltung. Stuttgart: Klett-Cotta.

Petzold, H. G. (2003). Integrative Therapie-Modelle, Theorien und Methoden für eine schulenübergreifende Psychotherapie(2.Aufl.). Paderborn: Junfermann.

Richter, K. F. (2011). Erzählweisen des Körpers. Göttingen:Vandenhoeck\&Ruprecht.

Rudolf, G. (2013). Strukturbezogene Psychotherapie. Leitfaden zur psychodynamischen Therapie struktureller Störungen (3. Aufl.).Stuttgart:Schattauer. 
Rudolf, G. (2019). Psychodynamisch denken - tiefenpsychologisch handeln. Praxis für tiefenpsychologisch fundierte Psychotherapie. Stuttgart:Schattauer.

Rudolf, G., Grande, T., \& Henningsen, P. (Hrsg.). (2008). Die Struktur der Persönlichkeit. Theoretische Grundlagen zur psychodynamischen Therapie struktureller Störungen (1.Aufl.). Stuttgart:Schattauer.

Sander, L. W. (1983). Polarity, paradox and the organizing process in development. New York: The Analytic Press, Taylor \&Francis Group.

Sreckovic, M. (Hrsg.). (2005). Lore Perls. Leben an der Grenze. Essays und Anmerkungen zur Gestalt-Therapie. Köln: Edition HumanistischePsychologie.
Stern, D. (2007). Die Lebenserfahrung des Säuglings (9. Aufl.). Stuttgart: Klett-Cotta.

Wöller, W. (2013). Trauma und Persönlichkeitsstörungen. Stuttgart: Schattauer.

Wöller, W., \& Kruse, J. (2015). Tiefenpsychologisch fundierte Psychotherapie. Basisbuch und Praxisleitfaden (4. Aufl.). Stuttgart: Schattauer.

Hinweis des Verlags Der Verlag bleibt in Hinblick auf geografische Zuordnungen und Gebietsbezeichnungen in veröffentlichten Karten und Institutsadressen neutral. 